\title{
Traumatismo craneoencefálico pediátrico
}

\section{Artículo de Revisión}

Nares López FE*, Terrones Saldívar MC**

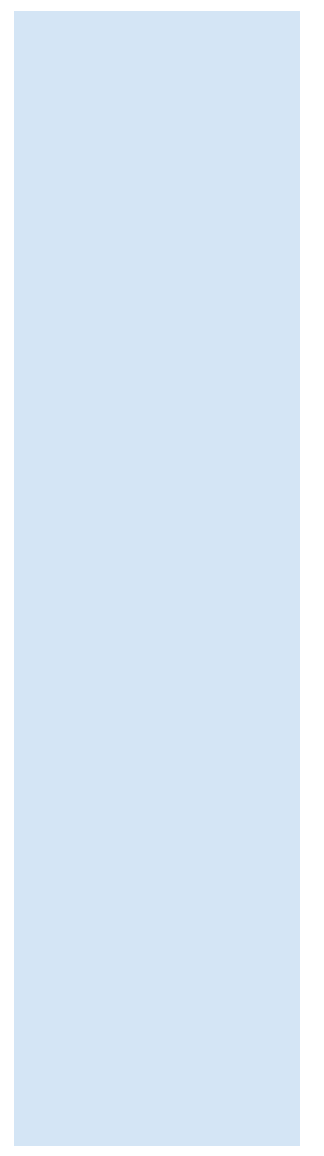

\section{Introducción}

El traumatismo craneoencefálico (TCE) puede definirse como cualquier lesión física o deterioro funcional del contenido craneal, secundario a un intercambio brusco de energía mecánica. Los traumatismos craneoencefálicos en niños son una causa frecuente de consulta en la emergencia pediátrica. El 75\% de todas las hospitalizaciones por traumatismos en los niños se deben a un TCE, al tiempo que el $70 \%$ de las muertes ocurren en las primeras 48 horas; $y$ se refiere una mortalidad que fluctúa entre el 20 y $35 \%$ 1,2. La mayor causa de morbimortalidad está relacionada con los accidentes de tránsito ${ }^{3} \mathrm{y}$ caídas ${ }^{1}$. Las lesiones intencionales, aunque en menor número, también contribuyen a incrementar las cifras del TCE, en la población infantil lo explica un aumento en el fenómeno del maltrato ${ }^{4}$ La gran mayoría fueron traumatismos craneoencefálicos leves $(79.2 \%)^{3}$.

A nivel internacional se estima que ocurren más de 5 millones de consultas en la emergencia ${ }^{3,5}$, entre 250 a 400.000 ingresos producto de TCE en Estados Unidos durante un año en pacientes entre los cero y catorce años con una mortalidad del $2,8 \%$ de los pacientes admitidos y un $11,6 \%$ de daño neurológico permanente en ese mismo grupo de pacientes ${ }^{1,3}$. Alrededor de uno de cada 500 niños en Estados Unidos padece de un TCE ${ }^{6}$. De los pacientes pediátricos atendidos con un TCE leve, sólo entre el 3 - 5\% tendrán un daño intracraneal y menos del $1 \%$ requerirá algún tipo de intervención neuroquirúrgica $1,7,8$

\footnotetext{
* Estudiante de Medicina del noveno semestre. Universidad Autónoma de Aguascalientes.

** Profesora Investigadora. Universidad Autónoma de Aguascalientes.
} 
Según cifras del SVELECE (Sistema de vigilancia Epidemiológica de lesiones por Causa Externa) de la Secretaría de Salud; los traumatismos craneoencefálicos constituyen la causa más frecuente de hospitalización en comparación con otras lesiones, en una proporción de 3:1. En México no se cuenta con la cifra exacta de mortalidad del traumatismo craneoencefálico severo (Escala de Coma de Glasgow < 8 puntos), sin embargo se estima de alrededor del $60 \%$ o más ${ }^{32}$.

La cifra de más del $60 \%$ de mortalidad es prácticamente de evolución natural. Los esfuerzos realizados hasta el momento, en México, no han resultado efectivos para reducir mortalidad del TCS a cifras cercanas a los estándares de calidad internacionales del $36 \% 32$.

| | | | | | | | | | | | | | | | | | | | | | | | | | | | | | | | | | | | | | | | | | | | | | | | | | | | | | | | | | | | | | | | | | | | | | | | | | | | | | | | | | | | | | | | | | | | | | | | | | | | | | | | | |

\section{Desarrollo \\ Fisiopatología}

\section{Contenido de la Bóveda Craneana:}

La bóveda craneana contiene 3 elementos principales que interactúan para mantener la presión dentro del sistema en un rango de $7-18 \mathrm{~cm} \mathrm{H} 2 \mathrm{O}(10-15 \mathrm{mmHg})$

- Cerebro: Comprende el $70 \%$ del volumen craneal, compuesto de agua en un $75-80 \%$. Divido en componentes intracelular y extracelular. El componente intracelular se subdivide a la vez en sustancia gris, un grupo celular denso poco distensible; y la sustancia blanca que es menos densa que la gris pudiendo almacenar incrementos de un 10\% o más de agua. El componente extracelular representa hasta el $20 \%$ del volumen cerebral. Puede existir reducción del volumen cerebral básicamente por la pérdida de agua extracelular. Sin embargo, a efectos de reducción o mantenimiento de la presión del sistema, este mecanismo compensador solo funciona en aumentos crónicos de presión no teniendo utilidad en los procesos agudos que ocurren producto de un $\mathrm{TCE}^{3}$.

- Líquido Cefalorraquídeo (LCR): Ocupa el $20 \%$ del volumen de la bóveda craneana. El volumen promedio en niños es de $90 \mathrm{ml}$, con una producción de 0,35 $\mathrm{ml} /$ minuto. En situaciones de aumento de la presión intracraneana, el LCR es el responsable de mantener una presión intracraneal dentro de límites normales desplazando su volumen hacia los espacios de reserva.

- Volumen Sanguíneo Cerebral (VSC): Espacio que ocupa el contenido sanguíneo dentro de la vasculatura cerebral. En condiciones ideales ocupa el $10 \%$ del volumen craneal. Es importante resaltar que el VSC no es directamente proporcional al flujo sanguíneo cerebral (FSC). En condiciones extremas, puede haber un aumento del VSC con un aumento de la presión intracraneana, y como resultante hay disminución del FSC. En condiciones de efecto de masa, existe el desplazamiento del VSC, en especial el de los senos venosos, en un intento de mantener la presión del sistema dentro de límites compatibles con la vida ${ }^{3}$.

\section{Continente de la Bóveda Craneana}

- Huesos craneales: Durante el primer año de vida, debido a la presencia de las fontanelas y la no consolidación de las suturas, puede haber un aumento de la circunferencia cefálica producto de un incremento de la presión intracraneal. Esto se observa con frecuencia en las hidrocefalias, o de manera aguda 
debido a un TCE. Este es un mecanismo compensatorio que evita un aumento brusco de la presión intracraneal, que pudiera poner en peligro la vida del niño. A partir de los 18 meses de vida se cierra la fontanela anterior, y el cráneo comienza a ser una caja rígida poco distensible ${ }^{3}$.

- Repliegues de duramadre: Esta forma compartimientos independientes que protegen al cerebro de desplazamientos masivos durante los golpes o desaceleraciones bruscas.

\section{Presión de Perfusión Cerebral (PPC) y Flujo Sanguíneo Cerebral (FSC).}

La PPC es la diferencia existente entre la presión arterial media, (PAM) y la presión intracraneana (PIC).

Dicha presión es la que mantiene el FSC adecuado para cubrir las demandas metabólicas del cerebro. No existe un valor predeterminado de la misma, pero una PPC > $40 \mathrm{mmHg}$ debe ser mantenida en niños con un trauma craneoencefálico, manejando valores entre 40-65 $\mathrm{mmHg}$ consideradas diferencias relacionadas con la edad ${ }^{33}$.

EI FSC es la cantidad de sangre que pasa por cada 100 gramos de tejido en un minuto.

FSC = PPC / RVC (Resistencia Vascular Cerebral). El valor en adultos es de $50 \mathrm{ml} /$ $100 \mathrm{gr} /$ minuto.

El cerebro postraumático tiene una significativa incidencia de vasoespasmo, que probablemente incremente la RVC y disminuya la PPC, produciendo isquemia 33

Es esencial entender que para mantener un FSC adecuado, tiene que mantenerse una PPC apropiada, la cual dependerá en situaciones críticas de la PAM.

\section{Daños que Ocurren en un TCE}

Daño Primario: Producido al momento del evento, siendo proporcional a la magnitud y duración de la fuerza aplicada ${ }^{3}$. Existen 2 mecanismos para la producción de dicho daño:
- Lesión directa al parénquima cerebral.

- Daño axonal difuso - producto de la elongación brusca de los tractos axonales de sustancia blanca durante una fuerza de desaceleración brusca, la cual puede llevar a la ruptura de los mismos con muerte celular. Este tipo de daño es irreparable y la intervención del médico no cambia el pronóstico producto de dicha lesión.

Daño secundario: Son todos aquellos eventos fisiopatológicos y bioquímicos que se generan una vez producido el daño primario, llegando a un pico a las 48 - 72 horas $^{3}$. Se pueden resumir en lo siguiente:

\section{1.- Eventos Sistémicos:}

- Hipoxia e hipoventilación - La hipoxia se define como apnea, cianosis, $\mathrm{PaO}_{2}$ $<60-65 \mathrm{mmHg}$ o una saturación de oxígeno $<90 \%$, debe ser identificada y corregida rápidamente debido a que es un potente vasodilatador cerebral. Puede presentarse de forma secundaria a una obstrucción de vía aérea de un paciente inconsciente, daño a nivel de centro respiratorio, o por daño directo del parénquima pulmonar. La hipoventilación se define como una frecuencia respiratoria inefectiva para la edad, respiración irregular, periodos frecuentes de apnea, o hipercapnia cuantificada ésta última puede aumentar el FSC hasta un $350 \%$ de lo normal ${ }^{9}$.

- Hipotensión - La hipotensión en niños se define como la presión sanguínea sistólica menor a la percentila 5 para la edad o a partir de la observación clínica de signos de choque. Producto de hipovolemia o vasodilatación periférica debido a una lesión medular. Ésta puede estimarse mediante la siguiente fórmula: $70 \mathrm{~mm} \mathrm{Hg}+(2 \times \text { edad en años })^{33}$. Estos tres eventos son de gran importancia ya que existe evidencia de una tasa de mortalidad mayor al $60 \%$ asociada a la hipotensión contra un $22 \%$ en aquellos 
que no la presentaron. Esta tasa se eleva al $85 \%$ al combinarse con hipoxia ${ }^{33}$. Cabe destacar que ocasionalmente se requerirá el apoyo de drogas vasoactivas a fin de optimizar la Presión de Perfusión Cerebral tras la reanimación hídrica en el área de emergencias.

2.- Eventos Intracraneales:

- Pérdida de la autorregulación del Flujo Sanguíneo Cerebral (FSC) - se pierde el mecanismo intrínseco que ajusta el flujo sanguíneo tanto a las demandas metabólicas.

- Ruptura de la Barrera Hematoencefálica (BHE) - las sustancias normalmente excluidas pasarían libremente al parénquima cerebral.

- Formación de Edema Cerebral. Existen tres tipos:

1. Vasogénico: Aquel producido por el aumento de permeabilidad del endotelio vascular. Hay un aumento del VSC. Se encuentra alrededor de tumores, abscesos, en hematomas intraparenquimatosos, meningitis y encefalitis. También se observa durante las primeras 48 horas de un TCE, llamado hiperemia cerebral, se encuentra preferentemente en la materia blanca ${ }^{10}$. Las neuronas no presentan un daño primario, y el edema no significa lesión axonal.

2. Citotóxico: Edema netamente celular producto del fallo del sistema de trasporte ATP dependiente de la membrana celular. Significa muerte celular, y no cambia con la terapéutica. Trabajos recientes sugieren que este tipo de lesión es la predominente en pacientes que experimentan un TCE ${ }^{10}$.

3. Intersticial: Es debido al aumento de la presión hidrostática del LCR. Se debe a un déficit de absorción del LCR, produciendo hidrocefalias.

Las ondas de choque que viajan a través del cráneo y del cerebro desde el impacto, así como los efectos de la inercia, resultarán en daño adicional. Los impactos en la región temporoparietal deforman más fácilmente al cráneo que los que suceden en las áreas frontal u occipital, ya que éstas requieren hasta el doble de fuerza para conseguirlo ${ }^{12}$.

\section{Concusiones}

Es la interrupción corta y transitoria de la función neurológica posterior a un trauma en cráneo clasificado como menor, usualmente como consecuencia de las fuerzas de aceleración-desaceleración localizadas en cabeza en niños menores. Los síntomas agudos incluyen agitación, letargia, confusión o irritabilidad. Las decisiones para el manejo deben basarse en las mismas que para el TCE leve ${ }^{10}$. No es necesario que exista pérdida de la conciencia para que ésta sea clasificada como concusión; sin embargo se describió que ocurre deficiencia significativas en la memoria, el aprendizaje y en la adquisición de nuevos conocimientos en estudiantes atletas jóvenes ${ }^{1}$.

\section{Síndrome del Segundo Impacto.}

Acuñado por Saunders y Harbaugh en 1984; ocurre cuando una persona sufre un segundo TCE cuando los síntomas del primero aún no se han mitigado. Ocurre comúnmente en atletas jóvenes. Este síndrome propicia una rápida cascada de eventos que culminan en congestión vascular cerebral, causando aumento de la PIC y herniación que conduce a la muerte. Su diagnóstico es infrecuente y raramente fatal ${ }^{10}$.

\section{Eventos inflamatorios}

Posterior al daño primario, se produce una activación de la cascada inflamatoria que lleva a una alteración de la microcirculación con fenómenos de isquemia - reperfusión y la formación de radicales libres. Estos radicales son los responsables de la peroxidación de las membranas lipídicas de neuronas, glías y células endoteliales, produciendo una 
reacción en cadena que a la final altera el flujo iónico celular con muerte de las mismas. También en este grupo están el glutamato y el aspartato, conocidos como neurotrasmisores excitatorios, los cuales producen edema citotóxico ${ }^{3}$.

\section{Síndrome de Hipertensión Intracraneana} Todas aquellas manifestaciones clínicas que se presentan cuando ocurre un aumento de la presión intracraneana. Como el sistema no permite incrementos importantes de volumen, debido a que está rodeado por los huesos craneales, cuando uno de los componentes aumenta de volumen, tiene que disminuir alguno de los otros dos para tratar de mantener una presión dentro de rangos fisiológicos.
La hipótesis Monro-Kellie propone que el incremento en alguno de los componentes debe ser compensado por el descenso de uno o más del resto de los componentes, para que el volumen total permanezca inalterado ${ }^{34}$.

EI LCR es el primer volumen a ser modificado, tendiendo a ser desplazado al principio desde el espacio subaracnoideo de la convexidad cerebral hacia el de la médula espinal. Cuando el volumen de LCR está totalmente desplazado hacia los espacios de reserva, la compliance o distensibilidad intracraneal está disminuida, lo que hace que cualquier aumento de volumen, por mínimo que sea, genere un importante aumento de presión dentro del sistema (volumen crítico).

Doctrina Monro-Kellie - Compensación Intracraneal para Masas en Expansión
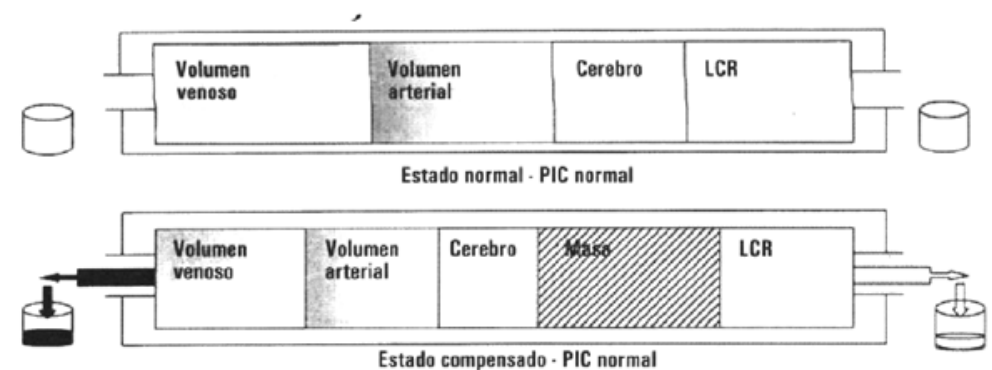

Estado compensado. PIC normal

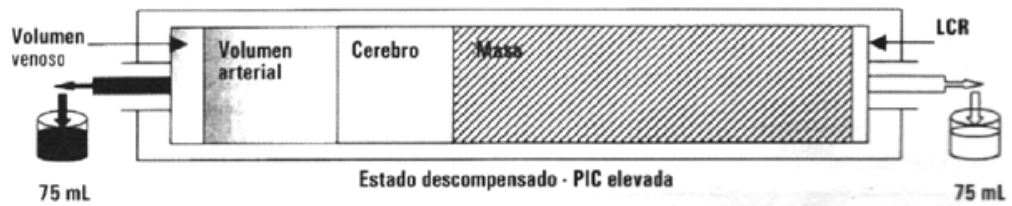

Por último, al ser usados todos los espacios de reserva y desplazado tanto el LCR como el VSC, el cerebro comienza a desplazarse por diferencia de presión a través de los repliegues de duramadre, (Falx cerebri y Tentorio), y a través del foramen magno, lo que es denominado herniaciones cerebrales ${ }^{3}$.
Tipo de Lesiones Encontradas.

- Hematoma Sub-galeal - colecciones de sangre entre el cuero cabelludo y los huesos craneales. No producen efecto de masa intracraneal. Debe tenerse precaución en los lactantes menores, ya que debido a la laxitud del cuero cabelludo, pueden producirse hematomas 
de gran tamaño que en ocasiones causan anemia e hipovolemia ${ }^{11}$.

- Laceraciones - heridas cortantes del cuero cabelludo, las cuales presentan sangrado profuso debido a la vascularidad de dicho tejido.

- Fracturas de Cráneo - pueden ser únicas o múltiples, lineales, con diástasis de sus bordes, fractura con hundimiento, y fractura de base de cráneo ${ }^{3,12}$. Las fracturas lineales sin desplazamiento de sus bordes son las más frecuentes y generalmente no requieren tratamiento, aunque indican que hubo una fuerza suficiente para poder producir un daño intracraneal. Las fracturas parietales a nivel de la arteria meníngea media pudieran producir sangrado de dicho vaso, lo cual produciría un hematoma epidural con un síndrome de hipertensión endocraneal. Las fracturas con hundimiento de las tablas óseas siempre requieren de consulta neuroquirúrgica, ya que son quirúrgicas hasta que no se demuestre lo contrario. Las fracturas de base de cráneo son difíciles de evidenciar sin embargo, existen signos clínicos que nos orientan hacia la misma: signo de mapache (fractura del techo de la órbita), signo de Battle (hemotímpano o hematoma a nivel de la apófisis mastoides del hueso temporal), rinorraquia, otorraquia.

- Hematomas intracraneales: Son los epidurales, subdurales, intraparenquimatosos y la hemorragia subaracnoidea.

- Hematoma Epidural: Colección de sangre ubicada entre la concavidad ósea y la duramadre. Generalmente son de origen arterial, (meníngea media), y su formación es rápida, dando manifestaciones clínicas durante las primeras horas posterior al traumatismo. La imagen tomográfica característica es la de un lente biconvexo ${ }^{3,13}$.

- Hematoma Subdural: Colección de sangre ubicada entre la duramadre y la corteza cerebral. Se forma debido a la ruptura de vasos venosos de baja presión que disecan la aracnoides de la duramadre coleccionándose en la convexidad craneal. Su presentación puede ser aguda, subaguda, (3-5 días), o inclusive crónica, (2 -3 semanas). La clínica observada en el niño es de cefalea, vómitos, alteración del humor y del estado de conciencia. Cuando se presentan en preescolares sin historia de traumatismo reciente, hay que considerar el diagnóstico del "Síndrome del Niño Batuqueado" por maltrato infantil. La imagen en la TAC en la fase aguda es el de una colección hiperdensa creciente generalmente a nivel parietal. En la fase subaguda y crónica la imagen se torna isodensa siendo más difícil de observar en una TAC sin contraste. Se recomienda realizar TAC de cráneo con contraste después de las 48-72 horas posterior al traumatismo ${ }^{3,13}$.

- Hematoma intraparenquimatoso: Colecciones de sangre dentro del parénquima cerebral, consecuencia de fuerzas traumáticas severas. Si las hemorragias son lo suficientemente grandes, producen efecto de masa con el resultante síndrome de hipertensión endocraneana. Se evidencian imágenes hiperdensas entre el tejido cerebral.

- Hemorragia subaracnoidea - es la presencia de sangre dentro del espacio subaracnoideo debido la ruptura de vasos piales. Ocurre como consecuencia de TCE severos de origen contuso, o penetrantes. Tomográficamente se observa como un líquido hiperdenso en áreas bañadas por el LCR.

\section{Clasificación Clínica}

La clínica observada está relacionada con los daños intracraneales producidos por un TCE, desde una discreta cefalea, posterior a un TCE leve, pasando posteriormente por 
vómito, pérdida transitoria de la conciencia, desorientación, confusión, amnesia, convulsiones, alteración y deterioro del estado de conciencia, hasta la focalización neurológica, coma y muerte observada en los TCE severos 3,10 .

Dependiendo del tipo de lesión encontrada, la clínica puede instaurarse en el momento del episodio, horas e inclusive días o semanas después del evento. La mayoría de las manifestaciones clínicas observadas en los TCE moderados y severos son la consecuencia de un síndrome de hipertensión intracraneana.

Lo importante de la clínica es orientar al médico a detectar tempranamente una lesión Intracraneal (LIC), que pueda poner en riesgo la vida del niño al no recibir tratamiento adecuado. Como la clínica varía de acuerdo a la severidad del caso, se ha realizado una clasificación de los TCE. Si bien no existe un consenso totalmente establecido, de acuerdo a la escala de Glasgow y a la clínica encontrada, los TCE se pueden clasificar en leves, moderados y severos $3,7,10$.

Escala de coma Glasgow modificada

\begin{tabular}{ccc}
\hline Puntaje & LACTANTE & ADULTO \\
& & Apertura ocular \\
& & Espontánea \\
\hline 3 & Espontánea & Al hablarle \\
\hline 2 & Al hablarle & Al dolor \\
\hline 1 & Al dolor & Sin respuesta \\
\hline & Sin respuesta & \\
\hline 6 & & Respuesta motriz \\
\hline 5 & Adecuado al hablarle & Adecuado al hablarle \\
\hline 4 & Al estímulo cutáneo & Al estímulo cutáneo \\
\hline 3 & Defensa al dolor & Defensa al dolor \\
\hline 2 & Flexión anormal & Flexión anormal \\
\hline 1 & Extensión anormal & Extensión anormal \\
\hline Sin respuesta & Sin respuesta \\
\hline 5 & & \\
\hline 4 & Balbuceo & Orientada y conversa \\
\hline 3 & Respuesta verbal & Desorientada-confusa \\
\hline 2 & Llanto al dolor & Palabras inadecuadas \\
\hline 1 & Quejidos al dolor & Sonidos inespecíficos \\
\hline
\end{tabular}

\section{Traumatismo Craneoencefálico Leve}

Cuando el paciente tiene un puntaje entre 15 y 14 puntos en la escala de Glasgow.

- Llegan a la emergencia en buenas condiciones.

- Discreta cefalea.

- Menos de tres episodios eméticos.

- No hay historia de pérdida de la conciencia o es menor de un minuto.

- No presentan déficit neurológico ni amnesia postraumática. 


\section{Traumatismo Craneoencefálico Moderado}

El paciente presenta un puntaje entre $13 y$ 9 puntos en la escala de Glasgow. Además del puntaje, se considera moderado cuando existe:

- Una pérdida de conciencia de 5 minutos.

- Amnesia postraumática.

- Tendencia a la somnolencia.

- Más de 3 episodios de emesis posterior al evento.

- Convulsiones postraumáticas.

- Signos de Fractura de base de cráneo.

- Pacientes politraumatizados con TCE.

- Pacientes con trauma facial importante.

Este mismo segmento puede ser subclasificado en:

- TCE Moderado: cuando tiene un puntaje de 13 y 11 puntos en la escala de Glasgow.

- TCE Moderado-Severo: Cuando hay más de 5 minutos de pérdida de conciencia, y un puntaje de 9 a11 puntos en la escala de Glasgow.

\section{Traumatismo Craneoencefálico Severo}

Cuando el paciente tiene un puntaje menor o igual de 8 puntos en la escala de Glasgow. Además de la escala se consideran severos:

- Cuando hay un descenso de 2 ó mas puntos en un paciente con un puntaje previamente mayor, que no sea causado por convulsiones, medicamentos, hipotensión arterial o factores metabólicos.

- Focalización neurológica.

- TCE penetrante.

- Hundimiento craneal visible o palpable.

- Fractura múltiple de cráneo.

- Maltrato infantil, Síndrome de Niño Batuqueado.

\section{Pérdida de la conciencia}

Considerado un predictor de lesión intracraneana en paciente pediátrico; se acepta que el riesgo de daño cerebral aumenta en pacientes pediátricos con historia de pérdida de conciencia o amensia. En un estudio, la letargia (o cualquier estado de conciencia alterado) resultó ser el signo o síntoma que mejor predijo la LIC $(78 \%)^{14}$. Ni la presencia ni la ausencia de pérdida de la conciencia está relacionada con los hallazgos tomográficos ${ }^{15}$.

\section{Manejo del Paciente en la Emergencia $^{3}$}

Mientras se está realizando la evaluación inicial del niño, es importante conocer:

1 Mecanismo del traumatismo.

2 Si hubo o no pérdida de conciencia posterior al mismo.

3 Presencia o no de convulsión postraumática.

\section{La Evaluación Inicial del niño debe de} constar:

$1 \mathrm{ABC}$

2 Signos vitales.

3 Valoración neurológica rápida por el sistema AVDS:

Alerta al momento de llegar.

Responde a Estímulos Verbales.

Responde a Estímulos Dolorosos.

Sin Respuesta a estímulos.

Es importante que en dicha evaluación esté incluida la hora de realización, ya que la misma nos servirá como base para observar la evolución del niño.

\section{La Evaluación Secundaria, tomando} como punto de partida un TCE aislado, debe constar de:

1 Signos vitales: FC, FR y PA que tenga la PAM. PAM = (PA sist+2PA diast) $/ 3$.

2 Determinación de signos de Fracturas de Cráneo: Hemotímpano, Signo de Batle, Signo de Mapache, otorraquia o rinorraquia, depresión de la bóveda craneana o lesión penetrante.

3 Evaluación Neurológica la cual debe constar:

a Escala de Glasgow. 
b Reflejo Pupilares. La alteración de los mismos es una medición indirecta de herniación y lesión de tallo cerebral. Se considera anisocoria cuando existe una diferencia pupilar mayor de un milímetro. Una pupila midriática es cuando la misma es mayor de cuatro milímetros al momento del examen. En la pupila fija hay menos de un milímetro de constricción al ser iluminada con linterna por el examinador ${ }^{16}$.

Un signo temprano de herniación del lóbulo temporal es la dilatación leve de la pupila con una respuesta lenta a la luz. Conforme la herniación empeora, hay mayor dilatación de las pupilas seguida de ptosis y paresia del músculo recto interno y de otros inervados por el III par. Ello resulta en exotropia del ojo, que es diagnóstica de la parálisis del III par ${ }^{17}$.

c Examen de Pares Craneales.

d Movimiento de las extremidades.

e Respuesta Plantar.

Finalmente, una vez valorado el paciente, se clasifica el TCE en leve, moderado o severo. Dicha clasificación nos sirve para clasificar el riesgo de LIC del paciente, nos orienta hacia los estudios radiológicos a realizar, la instauración de terapéutica de emergencia, el tiempo necesario de observación intrahospitalaria, y la pertinencia o no de trasladar al paciente hacia un centro que cuente con atención neuroquirúrgica. El manejo correcto de la decisión clínica puede disminuir la necesidad del uso de TAC de cráneo hasta en un $23 \%{ }^{7}$.

\section{TCE Leves}

Los pacientes con TCE leves no requieren de mayor evaluación paraclínica. Sólo un $3 \%$ de los pacientes se deterioran ${ }^{17,18}$. La observación debe ser ambulatoria, dándole al familiar instrucciones de acudir nuevamente a la emergencia si presenta clínica durante las primeras 72 horas después del evento (deterioro del estado de conciencia, cefalea continua, dificultad para despertarse, vómitos intratables, convulsiones, y algún cambio en la conducta del niño). El vómito postraumático no es un predictor independiente de una lesión intracraneal, este síntoma está más relacionado con la predisposición del huésped al vómito que a las lesiones que presenta al hablar de un TCE leve ${ }^{19}$. Su tratamiento es sintomático ${ }^{1,3}$.

Se debe administrar el toxoide tetánico en caso de presentar heridas abiertas ${ }^{17}$. Con respecto a la imagenología no existe un consenso generalizado. La mayoría escogió la observación ambulatoria como primera alternativa terapéutica, mientras que sólo un $1 \%$ escogió la tomografía y un 3\% escogió la radiología convencional como ayudas en el diagnóstico ${ }^{20}$. Algunos textos refieren que los lactantes menores con hematomas en cuero cabelludo ameritan radiografía de cráneo postero-anterior y lateral ${ }^{21}$.

De cualquier forma, a pesar de ser considerado un evento benigno, el TCE leve puede estar asociado a complicaciones a largo plazo ales como trastorno de stress post-traumático o síndrome post-concuso (cefaleas, trastornos de la memoria y la concentración, ansiedad, trastornos del ánimo), ambos frecuentemente relacionados con trastornos del sueño ${ }^{22}$.

\section{TCE Moderados}

Los hallazgos asociados con LIC son: signos de fractura de cráneo, pérdida de conciencia de más de cinco minutos, y alteración del estado de conciencia. Aproximadamente de un 10 a un $20 \%$ de los pacientes se deterioran hacia TCE severo ${ }^{18}$. Estos pacientes deben observarse por un mínimo de 6 horas para seguir su evolución. Si durante este lapso de tiempo no hay mejoría clínica, existen hallazgos clínicos asociados con LIC, o hay deterioro de su cuadro clínico, el paciente debe permanecer en el hospital por 24- 48 horas. 
Si bien el estudio radiológico de elección es una TAC de cráneo, se recomienda realizar de entrada la radiografía de cráneo PA y lateral y radiografía de columna cervical lateral a todos los pacientes que ingresen con este diagnostico. En caso de que se evidencien hallazgos asociados con LIC, fractura de cráneo en la radiografía o convulsión postraumática, es indispensable el estudio tomográfico ${ }^{3}$. La radiografía de cráneo solamente da información acerca de la estructura ósea, (continente), y es menos sensible y específica que la TAC craneal para detectar lesiones parenquimatosas. Es por eso que la TAC debe ser, en condiciones ideales, el estudio de elección en este tipo de paciente ${ }^{5,10}$.

Este grupo de pacientes deben tener una vía venosa tomada, mantenerse en ayuno absoluto mientras se observa su evolución, y una hidratación parenteral de mantenimiento.

\section{Convulsiones postraumáticas}

Los pacientes pediátricos tienen un riesgo mayor de presentar convulsiones tempranas comparado con adultos que presentan un TCE severo ${ }^{33}$.

Ocurren tanto como en un $12 \%$ de todos los pacientes en TCE no perforante y hasta en un $50 \%$ en los TCE perforantes ${ }^{10}$.

Tipos:

- Las convulsiones inmediatas ocurren durante los primeros segundos posterior al traumatismo producto de la despolarización brusca de la corteza cerebral, son generalizadas y de corta duración. Las mismas no repercuten sobre la evolución del niño.

- Las convulsiones tempranas son las ocurren durante la primera semana posterior al traumatismo, siendo más frecuentes durante las primeras 24 horas. Son secundarias a complicaciones intracraneales tales como hemorragias, edema cerebral o infarto cerebral.

- Las convulsiones tardías son las que ocurren una semana después de haber ocurrido el evento. Son producto de focos irritativos posteriores a la isquemia y cicatrización cerebral. Ocurren principalmente en niños con TCE severos en los cuales existe laceración de la duramadre, o hematomas intraparenquimatosos.

El tratamiento inicial para las convulsiones tempranas es con diazepam 0,4 mg/ $\mathrm{kg} /$ dosis IV, cada 5 minutos para un total de $20 \mathrm{mg}$ o por vía rectal a dosis de 0,5 $\mathrm{mg} / \mathrm{kg}$. Puede utilizarse también lorazepam 0.05 a $0.15 \mathrm{mg} / \mathrm{kg}$ IV, cada 5 minutos hasta un total de $4 \mathrm{mg}$ (más efectivo para el abordaje del estado epiléptico) ${ }^{10}$ Posteriormente, se usa la fenitoína o ácido valproico como medicamentos para evitar su recurrencia. El riesgo de epilepsia posterior a una convulsión postraumática temprana es de un $15 \%{ }^{3}$

El estudio de la Brain Trauma Foundation ${ }^{23}$ concluye que pudiera usarse el tratamiento anticonvulsivante profiláctico para prevenir convulsiones postraumáticas, en aquellos pacientes que presenten factores de riesgo asociados a convulsiones posterior al TCE. Dichos factores de riesgo son:

- Puntuación < 10 puntos en la ECG.

- Contusión cortical.

- Fractura con hundimiento craneal.

- Hematoma epidural y/o subdural, herida penetrante.

- Convulsión durante las primeras 24 horas posterior al evento.

La revisión del sistema Cochrane ${ }^{3}$ concluye que el uso profiláctico de anticonvulsivantes es efectivo para reducir las convulsiones postraumáticas tempranas, pero no hay evidencias de que reduzca la aparición de convulsiones tardías, ni que tenga efecto sobre la mortalidad o las secuelas neurológicas.

Las guías para el manejo del traumatismo craneoencefálico severo pediátrico no recomiendan el uso profiláctico de medicamentos anticonvulsivantes para prevenir convulsiones tardías. En el periodo agudo 
posterior a un TCE severo, las convulsiones incrementan las demandas metabólicas, aumentan la presión intrancraneana y pueden desarrollar una lesión cerebral secundaria. La prevención de las convulsiones tempranas ha sido sugerida para prevenir el desarrollo de epilepsia crónica. Por otro lado, los antoconvulsivantes han sido asociados a efectos adversos como dificultad para el aprendizaje, rash, anomalías hematológicas, ataxia y modificación en el comprotamiento ${ }^{33}$.

Como conclusión el uso de anticonvulsionantes en el TCE es controvertido; por lo que comúnmente solo se emplea en pacientes con crisis comprobadas o en aquellos sometidos a ventilación mecánica ${ }^{2}$.

Con respecto a los vómitos postraumáticos, los mecanismos precisos por los cuales se producen son desconocidos. Se postula la teoría que las fuerzas de desaceleración brusca son las causantes de los mismos, produciendo una deformación transitoria del tallo cerebral, con estimulación del centro del vómito a nivel de la formación reticular. Los mismos se presentan en un $12 \%$ de los TCE en niños, y la presencia de los mismos aumenta cuatro veces el riesgo relativo de encontrar una fractura de cráneo ${ }^{3}$.

\section{TCE Severos}

Los pacientes con TCE severo deben ser manejados en centros hospitalarios que cuenten con especialidad neuroquirúrgica, y unidad de Terapia Intensiva Pediátrica.

En la primera valoración, debido al estado de conciencia del niño, es de extrema importancia garantizar una vía aérea permeable. Se observa si existe un patrón respiratorio constante y efectivo, (buena expansibilidad torácica, y FR > $20 \mathrm{rpm}$ ). De ser así, se suministra oxígeno húmedo por mascarilla a un flujo mayor de 5 Lts/ min. De lo contrario, es necesario intubar al paciente para evitar la hipoxia, hipoventilación y la broncoaspiración del conteni- do gástrico. Las guías Internacionales de manejo del trauma craneoencefálico severo en pacientes pediátricos recomiendan el control de la vía aérea con un puntaje de Glasgow $\leq 8$ puntos para evitar hipoxemia hipercarbia y aspiración ${ }^{32}$. Previo a la misma, se administrará lidocaína a dosis de 1,5 $\mathrm{mg} / \mathrm{kg}$ VEV para evitar aumento de la presión intracraneal durante el procedimiento. En los pacientes que presenten riesgos de traumatismo de columna cervical, deben de intubarse manteniendo la cabeza y el cueIlo en posición neutral ${ }^{24}$. Una vez garantizada la vía aérea, se ventilará al paciente con resucitador manual, a una frecuencia respiratoria inicial de 20 respiraciones por minuto con $100 \%$ oxigeno.

El principal objetivo del manejo de la vía aérea es mantener PCO2 30-35 mmHG, y Sat $\mathrm{O} 2>95 \%$. Posteriormente, hay que garantizar una PPC $>50 \mathrm{mmHg}$. Para ello es indispensable mantener presiones arteriales dentro de límites normales para la edad del paciente, manteniendo la PAM $>70 \mathrm{mmHg}$. Si el paciente está hipotenso, se realizan expansiones con solución de Ringer o solución salina 0,9\% a $10-20$ $\mathrm{ml} / \mathrm{kg}$ hasta garantizar PA adecuadas para su edad. La restricción hídrica en este tipo de pacientes es controversial, ya que puede llevar a la hipotensión con la resultante isquemia cerebral ${ }^{3}$. Es por eso que una vez restaurado el volumen intravascular, se recomienda mantener el paciente normovolémico.

En este tipo de pacientes es importante la protección gástrica, debido a la formación de úlcera de stress. La ranitidina a dosis de 2 - $4 \mathrm{mg} / \mathrm{kg} /$ día C/6-8 horas ayuda a prevenirla.

La incidencia de la pérdida de la olfacción es del $13 \%$ posterior a un TCE leve, $19 \%$ en TCE moderado y $25 \%$ en un TCE severo. La pérdida del gusto es rara, solo se presenta en el $0.5 \%$ de los casos de TCE en general; pero asociada a la pérdida postraumática del olfato su frecuencia 
aumenta hasta 5-7\% . Existen diversos mecanismos a nivel local o neuronal, lo importante es como esto afecta el pronóstico ya que sólo el $30 \%$ de los pacientes mejora. La mejoría debe observase en un plazo no mayor a un año. Algunos autores sugieren que la administración de sulfato de zinc puede ayudar a mejorar el déficit ${ }^{25}$.

La prevalencia de trastornos del sueño fue significativamente mayor entre adolescentes que experimentaron un TCE leve comparado con los sujetos control (28\% versus $11 \%, \mathrm{P}<0.05)^{22}$.

\section{Monitorización Neuroinvasiva}

El monitoreo de la presión intracraneal genera información continua necesaria para elegir una secuencia terapéutica escalonada de menor a mayor intensidad o dirigida hacia un mecanismo fisiopatológico específico. Su práctica se ha generalizado en el manejo del traumatismo craneoencefálico grave, extendiéndose ampliamente a otros procesos neurológicos que cursan con deterioro de nivel de conciencia secundario a edema cerebral e hipertensión intracraneal como la hemorragia subaracnoidea y la hidrocefalia, y en menor medida en hemorragia intracerebral, infartos, tumores, control postoperatorio e infecciones como encefalitis, meningitis y cisticercosis ${ }^{34,35}$.

Se encuentra indicado el monitoreo de la presión intracraneal en pacientes pediátricos con traumatismo craneoencefálico severo, ya que éstos poseen un riesgo elevado de presentar hipertensión endocraneana. No es una indicación de rutina en TCE leve y moderado pero puede ser utilizada en caso de lesiones con presencia de masa demostrada por tomografía axial computada asociada a sedación, bloqueo neuromuscular o anestesia. Su uso en TCE severo se asocia a los mejores resultados clínicos ${ }^{33}$.

Debe colocarse un sensor intracraneal con el objetivo de conseguir determinar de forma continua la PIC y el registro de on- das, detectar la hipertensión intracraneal, valorar la relación directa que existe entre la manipulación del paciente y el aumento de la PIC.

Las medidas de registro pueden realizarse en 4 espacios intracraneales a elegir: epidural, subaracnoideo, intraparenquimatoso e intraventricular, siendo los dos últimos los más frecuentemente utilizados, cada uno con sus ventajas e inconvenientes. Puede realizarse también una fontanometría (en menores de 18 meses). Como norma general el catéter siempre se colocará en el lugar donde existe la lesión, en el caso de que la lesión sea bilateral o difusa, el catéter se colocará en el lado derecho ya que es el que recibe mayor drenaje venoso.

La monitorización de la presión intraventricular es considerada el estándar de referencia. Es la más fiable a la vez que permite la evacuación terapéutica de líquido cefalorraquídeo (en caso de hemorragia ventricular o hidrocefalia) ${ }^{35}$.

Sistemáticamente se indica la monitorización de la presión intraparenquimatosa. El monitoreo intraparenquimatoso tiene un riesgo de infección del $1 \%$, el cual aumenta progresivamente a partir del tercer día, es fácil de colocar, se puede colocar en reanimación indistintamente por un anestesiólogo o neurocirujano. Es un método fiable de medición de la presión local aunque puede generar falsas medidas si no se maneja adecuadamente. Es frágil y con un costo elevado ${ }^{33,34}$.

Ambos métodos de monitorización son adecuados y confiables para monitorizar la PIC ${ }^{33}$.

Los factores que modifican la PIC son:

- Variaciones en la elevación de la cabecera.

- Rotación cefálica.

- Hiperextensión del cuello

- Manipulación de la vía aérea

- Aseo con sedación-sin sedación 
Al tratarse de una técnica invasiva, la monitorización de la PIC no está exenta de riesgos como hemorragia, infección, malfuncionamiento y obstrucción o malposición. Aunque estas complicaciones raramente provocan secuelas prolongadas en los pacientes ${ }^{35}$.

La necesidad de monitorización del flujo sanguíneo cerebral tiene importancia debido a la presencia de isquemia cerebral y la elevada prevalencia de alteraciones de los mecanismos de control del flujo sanguíneo cerebral (FSC) en los pacientes con un TCE grave. El FSC puede estimarse a partir de la medición de las diferencias arterio-yugulares de oxígeno o de otras variables hemometabólicas derivadas de la oxihemoglobina a nivel del bulbo de la yugular ${ }^{35}$.

\section{Signos de Herniación Cerebral}

Los signos clínicos que nos orientan hacia una herniación cerebral pueden ocurrir en cualquier momento durante la evolución del paciente. Los signos de herniación transtentorial se manifiestan por deterioro progresivo de la conciencia no explicado por eventos sistémicos acompañado por:

- Anisocoria ipsilateral a la lesión la cual puede llegar a midriasis paralítica.

- Parálisis del III par craneal.

- Hipertensión.

- Hemiparesia contralateral a la lesión, que progresa hacia rigidez de descerebración.

- Bradicardia.

- Arritmia Respiratoria.

\section{Fenómeno de Cushing}

También llamado Reflejo de Cushing, es una entidad clínica que puede ocurrir durante la reanimación del paciente con un TCE severo. Incluye tres signos clínicos: ${ }^{8}$

1 Hipertensión severa progresiva

2 Bradicardia

3 Respiraciones errantes o irregulares Está directamente relacionado con incrementos en la PIC (33\%) y en la herniación cerebral. La presencia de éste requiere de un manejo rápido y agresivo que incluya hiperventilación y terapia osmótica máxima ${ }^{10}$.

Es por eso que deben realizarse monitorizaciones frecuentes, (cada 5 minutos), de los signos vitales y valoración neurológica anotando los resultados.

\section{Terapéutica de emergencia para el manejo de hipertensión intracraneana}

Definida como la elevación patológica de la presión intracraneal, debe de iniciarse cuando la PIC es $>20 \mathrm{mmHg}$ corroborada por una valoración clínica constante y datos de la presión de perfusión cerebral ${ }^{33}$.

1 Hiperventilación Mantener $\mathrm{PCO}_{2}$ en 30-35 mm H. La hiperventilación reduce la PIC al inducir hipocapnia; de esta manera se puede disminuir el volumen sanguíneo cerebral a través de la reducción del flujo sanguíneo cerebral por vasoconstricción. Como se mencionó, la hiperventilación prolongada no se recomienda porque provoca vasoconstricción e isquemia graves. La hiperventilación profiláctica tampoco está recomendada 8, 10,33.

2 Deshidratantes cerebrales Debe utilizarse manitol al 20\%: $0.25-1 \mathrm{gr} / \mathrm{kg} /$ dosis a pasar en 15-30 minutos, sin diluir ${ }^{2}$. Antes de su administración el paciente debe encontrarse estabilizado y euvolémico. El efecto terapéutico rápido de disminución de la viscosidad sanguínea y un efecto osmótico que se desarrolla en los siguientes 15 a 30 minutos, éste persiste por las siguientes 6 horas requiriendo de una barrera hematoencefálica intacta. Debe mantenerse la osmolaridad sérica por debajo de los $320 \mathrm{mOsm} / \mathrm{L}$. Puede considerarse también su uso en pacientes que muestran signos de herniación cerebral o deterioro neurológico agudo. El uso profiláctico de manitol no se recomienda ${ }^{33}$. No debe usarse si hay hipotensión por 
el peligro de desarrollar necrosis tubular aguda, o hay compromiso del volumen intravascular.

3 Furosemida $1 \mathrm{mg} / \mathrm{kg} /$ dosis 15 minutos posterior a la administración de manitol. Existe sinergismo entre el manitol y furosemida, dando mejor efecto la combinación de las drogas que separadas ${ }^{3,8}$.

4 Monitorización de la hemodinámica cerebral mediante la determinación de la saturación de oxígeno en la vena yugular interna $\left(\mathrm{SO}_{2} \mathrm{y}=55-75\right.$ vol\% $)$, la determinación de la diferencia arterioyugular de oxígeno $\left(\right.$ DayO $\mathrm{O}_{2}=\mathrm{SaO}_{2}-\mathrm{SyO}_{2}$ $\times 1,39 \times \mathrm{Hb} / 100 \mathrm{mg} / \mathrm{dL}$, cuyos valores normales se sitúan entre 4-9 vol\%), con los cuales se puede determinar la postura más conveniente en relación con el patrón intracerebral isquémico o hiperémico 26,27 .

5 Solución Salina Hipertónica (SSH) utilizada en el tratamiento de pacientes con una PIC elevada a concentraciones que varían entre un 3.1 y $23 \%$, debe utilizarse la dosis necesaria para mantener la PIC $<20 \mathrm{mmHg}$. La SSH causa expansión de volumen plasmático, reducción del vasoespasmo y disminución de la repuesta inflamatoria postraumática HTS a concentraciones entre $3.1 \%$ a $23 \%$. La osmolaridad puede ser tolerada hasta $360 \mathrm{mOsm} / \mathrm{L}$ incluso en combinación con manitol. La SSH parece ser más benéfica en TCE Pediátrico que en adultos $10,28,33$

6 La hipotermia leve $\left(32-34^{\circ} \mathrm{C}\right)$ ha demostrado ser neuro protectora; disminuye los niveles de aminoácidos excitatorios en la región del peritrauma así como en el LCR. Se ha visto disminución en el consumo de antioxidantes y en la peroxidación de lípidos posterior a un TCE en niños. También posee efectos antiinflamatorios al disminuir niveles de interleucina(IL)-1 en LCR y de IL-6 en plasma y niveles de prostanoides.
En tres estudios realizados existieron complicaciones como sangrados, sepsis y neumonía ${ }^{28}$. La hipertermia provoca un daño postraumático aun mayor por aumento en la respuesta fisiopatológica al daño ${ }^{33}$.

7 Drenaje ventricular externo puede considerarse como una opción en el manejo pacientes pediátricos con TCE severo cerrado en donde se presenta hipertensión intracraneana refractaria a tratamiento médico. Se utiliza en conjunto con la monitorización de la PIC generalmente ${ }^{33}$. Las infecciones como meningitis y ventriculitis pueden ser tan altas como del $22 \%$. El cambio riguroso o retiro del catéter a los cinco días como máximo debe realizarse dado el alto riesgo de infección del SNC asociado a su uso que clínicamente puede manifestarse como alteración de la conciencia, déficit neurológico focal o convulsiones no explicados por otra causa, rigidez de nuca y fiebre sin otro foco evidente ${ }^{36}$. El drenaje ventricular externo puede utilizarse junto con un drenaje lumbar, ya que alrededor del $30 \%$ del líquido cefalorraquídeo se encuentra en la médula espinal; se indica cuando no exsite obstrucción a nivel de cisternas o alguna masa ${ }^{33}$.

8 Craniectomía descompresiva reconocida desde principios del siglo XIX. Se recomienda la descompresión frontoparietotemporal unilateral o bifrontal según sea el caso dentro de las primaras 48 horas, una PIC $<40 \mathrm{mmHg}$ o que muestren signos de una herniación cerebral ${ }^{33}$. Estudios experimentales y clínicos demostraron la eficacia de estos métodos en el manejo del aumento de la PIC refractario a tratamiento médico; de cualquier forma, existen reportes de exacerbación del edema y hemorragias posteriores ${ }^{28}$.

9 Corticoesteroides su uso en la práctica habitual en nuestro medio, no está 
indicado. La dexametasona, esteroide usado con mayor frecuencia, ayuda a reducir el edema producto de la manipulación quirúrgica. Pero no tiene efecto en los edemas citotóxicos, (hipoxicoisquémico, metabólico), producto de los TCE. Los esteroides están asociados con un incremento del riesgo de infección. La evidencia disponible indica que los esteroides no disminuyen la PIC, ni mejoran el pronóstico en los pacientes con TCE severo ${ }^{3}$.

10 Dexanabinol canabinoide sintético no sicotrópico, antagonista no competitivo de receptores NMDA. Se ha demostrado que hidroxila radicales e inhibe el factor de necrosis tumoral después de un TCE. Se ha demostrado su eficacia en las primeras seis horas posteriores al TCE. Se observa reducción en la PIC y la hipotensión sistémica. Las propiedades antiexcitotóxicas, antioxidantes y antiinflamatorias son prometedoras como complemento en el manejo del paciente con TCE ${ }^{28}$.

11 Factor VIla Recombinante ha tomado interés su uso sobretodo en las hemorragias intracerebrales y extra-axiales. El FVIIr ha sido utilizado para tratar hemorragias en pacientes con hemofilia. Su uso debe de ser individualizado ${ }^{10}$.

Está reportado que el FSC durante las primeras 24 horas posteriores al trauma es menor a la mitad de lo normal. Puede utilizarse SSH o manitol como primara línea dependiendo del curso clínico del paciente ${ }^{33}$.

No se recomienda el esquema terapéutico manitol/furosemida en más de dos oportunidades. El uso seguido de dicho esquema puede traer hiperosmolaridad, hipovolemia, alteraciones electrolíticas y elevación de la PIC 3 .

En algunos pacientes con TCE moderados y en todos aquellos con TCE graves, se recomienda la monitorización continua de la PIC, cuyos valores normales dependen de la edad (recién nacido por debajo de 2 $\mathrm{mmHg}$, lactantes entre 3-6 mmHg y niños mayores entre $6-15 \mathrm{mmHg})^{2}$.

La tercera fase de terapia intensiva con monitoreo de la PIC, ha mostrado que esta última por si sola puede reducir la mortalidad desde el $36 \%$ hasta el $25 \%$, en países desarrollados ${ }^{32}$.

Todos los criterios para diabetes insípida deben de ser conocidos para administrar desmopresina. La administración en niños de 3 meses a 12 años de edad es de $0.3-3$ $\mu \mathrm{g}$ por día vía IV ${ }^{6}$.

Se menciona también la terapia de segunda línea o hipertensión intracraneana refractaria con pentobarbital en infusión intravenosa ya que disminuye la PIC al disminuir el metabolismo cerebral y el VSC por vasoconstricción. No existe evidencia suficiente que apoye el efecto neuroprotector adicional de los barbitúricos al inhibir la peroxidación lipídica mediada por radicales libres o la estabilización membranal ${ }^{33}$. Sus efectos adversos son bradicardia, hipotensión, hipotermia y suceptibilidad a infecciones ${ }^{6}$.

El uso de sedantes, analgésicos y bloqueadores neuromusculares para facilitar la ventilación mecánica y el manejo de la PIC aumentada debe ser reservado para situaciones específicas; la profundidad y duración del bloqueo neuromuscular debe estar correctamente monitorizada y optimizada. De igual forma la vasodilatación cerebral producto del efecto hipotensor de la sedación que al producir aumento del volumen cerebral incrementa la PIC ${ }^{33}$.

\section{Resumen del Manejo del paciente pediá- trico con TCE severo}

- Paciente con ECG $\leq 8$ donde ya se descartó manejo quirúrgico inmediato.

- Inserción de monitor de la PIC.

- Control de la PIC y PPC (control de la temperatura, evitar la obstrucción ve- 
nosa yugular y mantenimiento de una adecuada oxigenación arterial).

- En caso de hipertensión intracraneana, sedación y analgesia

- En pacientes euvolémicos, elevar la cabeza $30^{\circ}$ con monitoreo continuo de la PIC y PPC (considerar bloqueadores neuromusculares)

- Si la PIC permanece elevada, drenaje de líquido cefalorraquídeo con acceso ventricular

- Si es inefectivo, considerar terapia hiperosmolar (manitol vs. SSH)

- Si la terapia no es efectiva, aumentar el nivel de ventilación hasta obtener una $\mathrm{PaCO}_{2}$ de 30-35 mmHg (a su vez considerar la medición de FSC o saturación venosa yugular)

- En todo momento debe de tenerse en cuenta la posibilidad de una masa o lesión intracraneal. Repetir TAC

- Para hipertensión intracraneana refractaria a tratamiento, son recomendadas las terapias de segunda línea (drenaje lumbar, craniectomía, barbitúricos, hipotermia).

\section{Maltrato infantil}

Es un problema de salud pública en el mundo, ya que uno de cada 10 niños es víctima de violencia, $15 \%$ sufren lesiones graves, y uno de cada 1000 puede morir. En México, el Instituto Nacional de Pediatría reportó en la autopsia de un grupo de 53 niños con historia de maltrato, los siguientes hallazgos:

- Signos de impacto en la cabeza (85\%)

- Fractura del cráneo (36\%)

- Hematoma subdural (81\%)

- Hemorragia subaracnoidea (48\%)

- Contusión (9\%)

- Hemorragia intracerebral(5\%)

- Lesión axonal difusa (5\%).

Microscópicamente se observaron datos de encefalopatía hipóxico-isquémica
(77\%) y la mortalidad se explicó por edema cerebral $(82 \%)$

La única forma de evitar los mecanismos de lesión primaria es fomentar entre la comunidad la cultura de prevención, con la concientización acerca de las medidas de seguridad, ya que con ello podrían evitarse hasta nueve de cada diez "accidentes" 4.

\section{El Principio Kennard y la Plasticidad neuronal}

Propuesto en 1930 a partir de investigaciones en el comportamiento de chimpancés infantes y adultos posterior a un daño cortical; término utlizado para reflejar la idea de que la recuperación de un daño cerebral era mejor en un cerebro en desarrollo que en uno ya desarrollado. En los últimos años este principio ha sido apoyado por la evidencia de mecanismos de plasticidad neuronal o la capacidad para reparar durante el desarrollo. De cualquier forma el Principio de Kennard no ha sido aceptado completamente; existen ciertos tipos de daño cerebral donde es más severo si se presentan a la edad infantil que a edad adulta dependiendo de su localización y la edad en que se presenta la lesión, tal es el caso de la corteza cerebral que posee el potencial más grande de plasticidad postlesional en niños debido a su capacidad de compensación interhemisférica e iontrahemisférica. Por otro lado en la corteza prefrontal suelen exsistir errores debido a que ciertas rutas neurales se desconectan prematuramente. Se concluye que el estado del neurodesarrollo juega un rol crucial en la determinación de la evolución de la lesión cerebral ${ }^{29}$.

\section{Marcadores Serológicos Pronósticos}

Incialmente se estudió para buscar, estandarizar y mejorar el manejo en emergencias con pacientes que padecieron un TCE leve. Se mencionan la enolasa neuronal 
específica, la creatin-kinasa BB, proteína básica de mielina y el S-100b.

La proteína tau también ha sido intensamente investigada; ésta posee una estructura microtubular y está localizada en los axones del sistema nervioso central (SNC), la proteína tau también estabiliza los microtúbulos que intervienen en el transporte celular e interactúa en la formación del citoesquelético. El daño hipóxico o trauma directo al axón causa que la proteína tau se vuelva extracelular. El estudio demostró una relación entre la concentración sérica de proteína tau y la severidad del trauma, sin embargo ésta diferencia no fue significativa, al igual que al correlacionarla con la TAC de cráneo, edad, sexo, o mecanismo del trauma, ECG, síntomas o examen físico ${ }^{30}$.

\section{Pronóstico Neurológico}

Es importante mencionar cuántos de los sobrevivientes consiguen la óptima reincorporación funcional sociofamiliar y escolar; ya que es precisamente ahí donde cobra importancia la investigación médica con aportaciones que beneficien el pronóstico neurológico y funcional del paciente que sufre de trauma craneoencefálico y que no muere, y combatir lo que en la actualidad surge como "el fracaso del éxito".

Se estima que entre el $21-42 \%$ de los niños con TCE severo desarrollarán una elevación de la presión intracraneal indetectable a pesar del manejo médico y quirúrgico adecuado. La tasas de mortalidad reportadas cuando la PIC es $>40 \mathrm{~mm} \mathrm{Hg}$ son de $29-100 \%$ a pesar de la terapia ${ }^{33}$.

En México el SVELECE reporta que el $31 \%$ de los pacientes con TCE mueren antes de llegar al hospital y el $30.1 \%$ muere dentro del hospital, siendo dados de alta solamente el $23.9 \%$. De los sobrevivientes, sólo el $16.2 \%$ se encuentran neurológicamente sanos y los demás quedan con secuelas tardías importantes ${ }^{32}$.
La publicación original de la Escala de Resultado de Glasgow (GOS) en 1975 fue con el fin de describir a los sobrevivientes de un TCE severo. Posteriormente la escala se amplió debido a su falta de sensibilidad a cambios funcionales significativos.

La escala describe la función social en general más que el déficit neurológico, y es muy útil en el monitoreo del paciente en recuperación. Debe realizarse a los seis meses ya que estudios muestran que la mayoría de los pacientes han alcanzado su categoría final dentro de la escala; esto no significa que el paciente no continúe mejorando a través de los años. Se considera un buen intervalo de seguimiento exitoso y es muy utilizada como medida de valoración del pronóstico neurológico ${ }^{37}$. La escala que se muestra a continuación:

\section{Extended Glasgow Outcome Scale (GOSE)}

\section{Muerte}

2 Estado vegetativo

3 Dependencia completa de otros

4 Dependencia de otros para algunas actividades

5 Incapacidad para participar en actividades sociales

6 Participación reducida en actividades sociales

7 Buena recuperación con déficit mental y social leve

8 Buena recuperación sin déficit

Otra manera de categorizar la severidad de la lesión y su efecto en el primer año después del evento traumático es mediante la escala KOSCHI (King's Outcome Scale for Childhood Head Injury), la cual se describe a continuación. 


\begin{tabular}{|ll|}
\hline \multicolumn{1}{|c|}{ King's Outcome Scale for Childhood Head Injury (KOSCHI) } \\
\hline 1 & Muerte \\
\hline 2 & $\begin{array}{l}\text { ESTADO VEGETATIVO } \\
\text { Incapaz de actuar recíprocamente con el ambiente }\end{array}$ \\
\hline 3 A & $\begin{array}{l}\text { INCAPACIDAD SEVERA } \\
\text { Capaz de seguir órdenes / incapaz de vivir de forma independiente }\end{array}$ \\
\hline 3 B & $\begin{array}{l}\text { INCAPACIDAD SEVERA } \\
\text { Capaz de seguir órdenes / limitado para vivir de forma independiente }\end{array}$ \\
\hline 4 A & $\begin{array}{l}\text { INCAPACIDAD MODERADA } \\
\text { La mayor parte independiente para la vida diaria, pero necesita un grado de supervisión para los } \\
\text { problemas físicos o de comportamiento. Tiene problemas abiertos. Puede estar en un programa } \\
\text { educativo especializado o de rehabilitación o requerir ayuda especial en la escuela. Los problemas de } \\
\text { comportamiento pueden haber excluido al paciente de la escuela. }\end{array}$ \\
\hline 4 B & $\begin{array}{l}\text { INCAPACIDAD MODERADA } \\
\text { Independiente para la vida diaria con respecto a su edad, pero con secuelas neurológicas que afectan } \\
\text { con frecuencia su vida cada día, incluyendo dificultades del comportamiento y de aprendizaje. Puede } \\
\text { también tener dolores de cabeza frecuentes. Puede estar en la escuela con o sin ayuda especial. }\end{array}$ \\
\hline 5 A & $\begin{array}{l}\text { Parece tener una recuperación funcional completa, pero tiene patología residual atribuible al TCE. Puede } \\
\text { sufrir dolores de cabeza que no afectan la escuela o la vida social. }\end{array}$ \\
\hline 5 B & \begin{tabular}{l} 
Sin secuelas \\
\hline
\end{tabular} \\
\hline
\end{tabular}

\section{Conclusiones}

El traumatismo craneoencefálico se origina de la energía mecánica ejercida sobre la cabeza; los cambios iniciales causados por esta energía se conocen como lesión primaria o mecánica, mientras que los eventos que le siguen y continúan causando más daño constituyen la lesión secundaria o no mecánica, ambas dan por resultado el daño final.

Los niños con traumatismo craneoencefálico severo tienen mayor probabilidad de supervivencia si son atendidos en centros de trauma especializados equipados con recursos técnicos y personal médico bien capacitado ${ }^{33}$.

En niños con traumatismo craneoencefálico, la medición de la presión intracraneal y la Escala de Coma Glasgow son los datos de mayor valor pronóstico ${ }^{31}$.

Investigaciones a futuro utilizando la neuroimagen funcional como la resonancia magnética funcional (RMIf) o la tomografía computarizada por emisión de fotón único (SPECT) se utilizarán como apoyo para comprender la reorganización funcional y las consecuencias de la lesión cerebral. Esto permitirá dictar mejores decisiones y programas de rehabilitación para maximizar la recuperación del paciente pediátrico ${ }^{4}$.

La única forma de evitar los mecanismos de la lesión primaria es fomentar, entre la comunidad, la cultura de prevención, con la concientización acerca de las medidas de seguridad. Así como promover programas que permitan identificar el maltrato infantil, y disminuir los factores de riesgo que lo favorecen ${ }^{4}$.

"Deben promoverse y practicarse estrategias para le prevención del TCE en niños al enseñar el ser precavido y hacer lo posible por enseñarles a medir riesgos... "Think First" 6. 


\section{Bibliografía}

1 Mary L. Thiessen, MD, Dale P. Woolridge, MD, PhD. Pediatric Minor Closed Head Injury. Pediatr Clin N Am 5320061 - 26

2 A.J. Lacerda-Gallardo, D. Abreu-Pérez. Traumatismo craneoencefálico en pediatría. Nuestros resultados. Rev Neurol 2003; 36 (2): 108-112

3 Azpurua Eraso, Luis. Traumatismo Craneoencefálico en Niños. Hospital Pérez de León de Petare.

4 Garduño-Hernández, Florentino. Traumatismo craneoencefálico en niños. Mecanismos de la lesión primaria Bol Med Hosp Infant Mex, 2008; 65.

5 Swaiman, Kenneth F. "Neurología Pediátrica Principios y Prácticas", 2da. Edición, Ed. Mosby, España 1996 págs. 971-985

6 Walter J. Faillace, MD, FACS. Management of childhood neurotrauma. Surg Clin N Am 822002 349-363.

7 Micelle J. Haydel, MD, Amit D. Shembekar, MD Prediction of Intracranial Injury in Children Aged Five Years and Older With Loss of Consciousness After Minor Head Injury Due to Nontrivial Mechanisms. annals of emergency medicine 42:4 october 2003; 42:4

8 Rubiano, Andrés "Neurotrauma y Neurointensivismo". Ed. Distribuna, Colombia 2008 pags 157-172

9 Poss WB, Brockmeyer D, Clay B, Dean JM. Patophysiology and management of the Intracraneal Vault. In Rogers M. Textbook of Pediatric Intensive Care Third Edition Williams \& Wilkins Baltimore 1996:645-66.

10 William Heegaard, MD, MPH, Michelle Biros, MD, MS. Traumatic Brain Injury. Emerg Med Clin N Am 2007 655-678.

11 Anton j, Pineda V, Martin C, Artigas J, Rivera J. Posttraumatic subgaleal hematoma: a case report and review of the literature. Pediatr Emerg Care 1999;15(5):347-9.

12 Volpe, Joseph J. "Neurología del Recién Nacido" 4ta. Edición, McGrawHill, EE.UU. 2003 págs. 867-873

13 Oski N, "Compendio de Pediatría", 2da. Edición, McGrawHill, EE.UU. 2006; 234-237.

14 Shane SA, Fuchs SM. Skull fractures in infants and predictors of associated intracranial injury. Pediatr Emerg Care 1997;13(3):198- 203.

15 Gruskin KD, Schutzman SA. Head trauma in children younger than 2 years: are there predictors for complications? Arch Pediatr Adolesc Med 1999;153:15-20.

16 Greenes DS, Madsen JR. Neurotrauma. In Fleisher G, Ludwig S Textbook of Pediatric Emergency Medicine 4th Edition. Lipincott Williams \& Wilkins Philadelphia 2000:1271-96

17 Noemí Padilla Campos, José Monge Margalli. Traumatismo craneoencefálico. Manejo en urgencias. TRAUMA, 2002; (5) 3: 92-99.

18 Intracranial Pressure. Neurotrauma. In http://www. trauma.org/neuro/icp.html Julio 2003

19 Liviana Da Dalt, Md, Barbara Andreola, Md, Paola Facchin, Md, Marzia Gregolin, Md, Andrea Vianello, Md, And Pier Antonio Battistella, Md. Characteristics of Children with Vomiting after Minor Head Trauma: A CaseControl Study. The Journal of Pediatrics • March 2007

20 Aitken ME, Fereiras CT, Davis R, Bell HS, Coombs JB, Kleinman LC, Homer CJ. Minor Head Injury in Children: current management practices of pediatricians, emergency physicians and family physicians. Arch Pediatr Adolesc Med 1998 Dec;152(12):1176-80.

21 Casado J, Martinez A. Traumatismo craneoencefálico en niños. En Casado J, Serrano A. Urgencias y Tratamiento del Niño Grave. Ediciones Ergon S.A. Madrid 2000:327-33.
22 Giora Pillar, Eran Averbooch, Neri Katz, Nir Peled, Yuval Kaufman, and Eli Shahar. Prevalence and Risk of Sleep Disturbances in Adolescents After Minor Head Injury. PEDIATRIC NEUROLOGY Vol. 29 No. 2

23 The Role of Antiseizure Prophylaxis following Head Injury. In Management and Prognosis of Severe Traumatic Brain Injury. A joint project of the Brain trauma Foundation and American Association of Neurological Surgeons, joint section of Neurotrauma and Critical Care. Brain Trauma Foundation 2000:159-165.

24 Advanced Airway Management: Rapid Sequence Induction for Emergency Intubation. In APLS The Pediatric Emergency Medicine Course. American College of Emergency Physicians and American Academy of Pediatrics. 3rd Edition Illinois 1998.

25 Evan R. Reiter, MD,Laurence J. DiNardo, MD, FACS Richard M. Costanzo, PhD Effects of head injury on olfaction and taste. Otolaryngol Clin N Am 37 2004, 11671184.

26 Pleguezuelo-Rodríguez EM, Quirós-Vigueiras O, RodríguezBencomo D, Montejo-Montejo J, López-Delgado $\mathrm{H}$. Monitoreo neurointensivo en pediatría. Generalidades. Rev Cub Pediatría 2001; 2: 115-22.

27 Kiening KL, Unterberg AW, Bardt TF, Schneider GH, Lanksch WR. Monitoring of cerebral oxygenation in patients with severe head injuries: brain tissue $\mathrm{PO} 2$ versus jugular vein oxygen saturation. J Neurosurg 1996; 85: 751-7

28 Hülya Bayır, MD; Robert S. B. Clark, MD; Patrick M. Kochanek, MD. Promising strategies to minimize secondary brain injury after head Trauma. Crit Care Med 2003 Vol. 31, No. 1 (Suppl)

29 Suzanne Munson, Elizabeth Schroth and Monique Ernst. The Role of Functional Neuroimaging in Pediatric Brain Injury. Pediatrics 2006;117;1372-1381. DOI: 10.1542/ peds.2005-0826

30 Cemil Kavalci MDa, Murat Pekdemir $\mathrm{MDa}_{1}{ }^{*}$, Polat Durukan MDa, Necip Ilhan PhDb, Mustafa Yildiz MDa, Selami Serhatlioglu MDc, Dilara Seckin MD. The value of serum tau protein for the diagnosis of intracranial injury in minor head trauma. American Journal of Emergency Medicine 2007 25, 391-395.

31 Sánchez RJJ y col. Indicadores en el pronóstico de niños con trauma craneoencefálico severo en una unidad de terapia intensiva Rev Mex Pediatr 2008; 75(4); 151 154

32 Murillo Tostado, C. "Sistemas Regionales de Trauma Craneal", Sociedad Mexicana de Cirguía Neurológica, sección neurotrauma 2006; 8:1-9.

33 The World Federation of Pediatric Intensive and Critical Care Societies. Guidelines for acute medical management of severe traumatic brain injury in infants, children and adolescent. Pediatric Critical Care Medicine 2003; 4:1-75

34 García, J.*. Actuación de enfermería en la monitorización de la presión intracraneal (P.I.C.). Enfermería Integral / Junio 2006

35 Protocolo de la Monitorización de la Presión Intracraneal y del Flujo Sanguíneo Cerebral. Sociedad Boliviana de Neurologia

36 Pedro Grille*, Gonzalo Costa., Alberto Biestro, Saúl Wajskopf§. Manejo del drenaje ventricular externo en launidad de cuidados intensivos. Guía práctica. Rev Med Urug 2007; 23: 50-55. Montevideo, Uruguay.

37 Bryan Jennett, MD, FRCS. Development of Glasgow Coma and Outcome Scales. Nepal Journal of Neuroscience 2:24-28, 2005. 